Check for updates

Cite this: RSC Adv., 2019, 9, 16690

\title{
Performance enhanced electromagnetic wave absorber from controllable modification of natural plant fiber $\dagger$
}

\author{
Lin Guo, ${ }^{a}$ Qing-Da An, (D) *a Zuo-Yi Xiao, ${ }^{a}$ Shang-Ru Zhai, (iD *a Li Cui ${ }^{a}$ \\ and Zhong-Cheng $\mathrm{Li}^{\mathrm{BD}}{ }^{\mathrm{b}}$
}

Short, surface rough carbon rods, which were derived from natural sisal fiber and went through two different modifications, with excellent electromagnetic wave absorption performance, were studied in this work for the first time. The structure-property relationship was clearly established here. It was shown that these green, cheap and easily obtained carbon rods with mass preparation possibility presented eye-catching absorbing behaviors towards electromagnetic wave. Based on the natural structure of sisal fiber, the minimum reflection loss of $\mathrm{KOH}$ activated product reached $-51.1 \mathrm{~dB}$ and the maximum effective absorbing bandwidth achieved $7.88 \mathrm{GHz}$. The magnetically modified sample presented $-48.6 \mathrm{~dB}$ of minimum reflection loss and $4.32 \mathrm{GHz}$ of optimal absorbing bandwidth. Its pioneering application in this field not only opens a new road for this traditional textile sisal fiber but also would possibly make a referable contribution to the design and synthesis of superior carbonaceous electromagnetic wave absorption materials based on bioresource.

Received 12th April 2019

Accepted 23rd May 2019

DOI: 10.1039/c9ra02764e

rsc.li/rsc-advances shortcomings such as expensive starting materials, fussy synthesis methods, harmful by-products, the further development of these materials in practical application has been limited. Thus, developing cheap, easy-obtained starting materials, accompanied with facile preparation and even mass production possibility is of important practical significance.

Biomass sources draw more and more attention when fossil energy dried up. Biomass carbon, a green and sustainable substitute of the traditional carbon, has natural advantages such as intrinsic porous structure, artificially tailored decoration and trace inherent metal ions including $\mathrm{Na}^{+}, \mathrm{K}^{+}, \mathrm{Ca}^{2+}$ and so on. ${ }^{14,15}$ More importantly, it offers advantages in mass preparation and production. Given this, many researchers, in particular for the EMW absorption investigators, pay attention to the development and application of these advanced sources. Singh and co-workers developed interesting heteroatom-doped carbon derived from chicken feather fibers, the inherent large fraction of heteroatoms and defects play a vital role in the outstanding performance, but the absorbing bandwidth was limited in $\mathrm{X}$ band $(8.2-12.4 \mathrm{GHz}) .{ }^{16}$ Wang's group prepared ferromagnetic hierarchical carbon nanofiber bundles derived from natural collagen fibers, apart from the complicated procedure, the starting materials were sundry and expensive. ${ }^{\mathbf{1 7}}$ On this occasion, the exploration of a more sustainable strategy to fabricate easy-obtained, low-cost and high-efficiency microwave absorption materials with broad absorbing band, even feasible mass production, is of practical significance to meet the requirement of ideal EMW absorbers. However, till now, there are limited successful examples accessible.
${ }^{a}$ Faculty of Light Industry and Chemical Engineering, Dalian Polytechnic University, Dalian 116034, P. R. China.E-mail: anqingdachem@163.com; zhaisrchem@163.com ${ }^{b}$ Key Laboratory of Optic-electric Sensing and Analytical Chemistry for Life Science, $M O E$, College of Chemistry and Molecular Engineering, Qingdao University of Science and Technology, Qingdao 266042, P. R. China

$\dagger$ Electronic supplementary information (ESI) available. See DOI: 10.1039/c9ra02764e 
We pursue the functionalization of materials by various preparation or regulation methods, but at the same time, we tend to ignore the cost of raw materials. Mother nature always gives, inspired by the concept of green chemistry, here, for the first time, we developed a novel EMW absorber which derives from cheap plant sisal fiber, the fabrication process is truly brief and the product obtained by two different modification methods all displayed brilliant performance. To the best of our knowledge, sisal fiber has been used in textile long ago, but almost no related works showing that it has directly applied breakthrough in the field of functional materials. Elammaran and his workmates gave a new investigation of sound absorption on sisal fiber poly composites, in which the voids and roughness of sisal fiber tremendously enhance the absorption coefficient. ${ }^{18}$ Up to now, sisal fiber has never been used in EMW absorption. As a groundbreaking, we obtained porous carbon rods from sisal fiber in different fabricating process, and then studied the structure-property relationship in EMW absorption. The as-made sample activated by $\mathrm{KOH}$ presents outstanding absorbing behavior, of which the minimal reflection loss is $-51.1 \mathrm{~dB}$ at $9.44 \mathrm{GHz}$ for $2.0 \mathrm{~mm}$ thickness and the effective absorbing bandwidth under $-10 \mathrm{~dB}$ climbs to 7.88 $\mathrm{GHz}$ from 10.12 to $18 \mathrm{GHz}$, corresponding with the thickness of $2.5 \mathrm{~mm}$. For the sample loaded with magnetic $\mathrm{Fe}_{3} \mathrm{O}_{4}$ particles, its performance is also impressive. The optimal effective absorbing bandwidth reaches $4.32 \mathrm{GHz}$ and the minimal reflection loss is $-48.6 \mathrm{~dB}$ at $10 \mathrm{GHz}$. Based on the eye-catching starting material and splendid performance of obtained product, we consider this work would possibly make a referable contribution to the design and fabrication of high-performance EMW absorption materials, especially open a new horizon of the biomass-derived absorbers.

\section{Experimental section}

\section{Raw materials}

Commercial natural sisal fiber was purchased from Guangxi Longli Plant Fiber Co. Lt. Hydrochloric acid ( $\mathrm{HCl}$ ), potassium hydroxide $(\mathrm{KOH})$, ferric chloride $\left(\mathrm{FeCl}_{3} \cdot 6 \mathrm{H}_{2} \mathrm{O}\right)$ and alcohol were all provided by Aladdin Industrial Co. Lt. The chemicals used in this work were of analytical grade without any further purification.

\section{Preparation of carbon rods}

$8 \mathrm{~g}$ natural sisal fiber was cleaned by distilled water and alcohol, and afterwards dried in oven at $60^{\circ} \mathrm{C}$ for $6 \mathrm{~h}$. After that, the fiber was transferred into a tube furnace for pre-carbonization process under $\mathrm{N}_{2}$ atmosphere, during which the heating rate was $5{ }^{\circ} \mathrm{C} \mathrm{min}^{-1}$ from room temperature to $300{ }^{\circ} \mathrm{C}$ for $2 \mathrm{~h}$. Then, the black hair-like sample was cooled down to room temperature naturally in tube furnace. Next, $3 \mathrm{~g}$ pre-carbonized sample was impregnated in $4 \mathrm{~mol} \mathrm{~L}^{-1} \mathrm{KOH}$ solution under magnetic stirring for $6 \mathrm{~h}$ and then re-carbonized in tubular furnace after drying. The carbonization condition was $800{ }^{\circ} \mathrm{C}$ for $2 \mathrm{~h}$ and the heating rate was $5{ }^{\circ} \mathrm{C} \min ^{-1}$. Finally, after water cleaning, drying and grinding, activated carbon rods were obtained and marked as ACR. The mechanism of $\mathrm{KOH}$ activation on carbon matrix can be illustrated as: ${ }^{19}$

$$
\begin{gathered}
6 \mathrm{KOH}+2 \mathrm{C} \rightarrow 2 \mathrm{~K}+3 \mathrm{H}_{2}+2 \mathrm{~K}_{2} \mathrm{CO}_{3} \\
\mathrm{~K}_{2} \mathrm{CO}_{3} \rightarrow \mathrm{K}_{2} \mathrm{O}+\mathrm{CO}_{2} \\
\mathrm{CO}_{2}+\mathrm{C} \rightarrow 2 \mathrm{CO} \\
\mathrm{K}_{2} \mathrm{CO}_{3}+2 \mathrm{C} \rightarrow 2 \mathrm{~K}+3 \mathrm{CO} \\
\mathrm{K}_{2} \mathrm{O}+\mathrm{C} \rightarrow 2 \mathrm{~K}+\mathrm{CO}
\end{gathered}
$$

The preparation process of magnetic modified samples was similar to that of ACR except the impregnation process using $1 \%(\mathrm{w} / \mathrm{v}) \mathrm{FeCl}_{3}$ solution, and the sample was labeled as FCR. For comparison, the sample only with one step carbonization and no modification was marked as CR. After removal of intrinsic metal ions that coming from raw fiber by acid pickling $(\mathrm{pH}=2$ $\mathrm{HCl}$ solution) for $\mathrm{CR}$, the obtained special sample was named as PCR. PCR would perform a simple comparison with CR in subsequent experiments to verify whether the inherent trace metal ions in sisal fiber might do something on EMW absorption. The main manufacturing process of ACR was illustrated in Scheme 1.

\section{Characterizations}

The micro-morphologies of samples were investigated by fieldemission scanning electron microscopy (SEM, JEOL JSM-7200F) and transmission electron microcopy (TEM, JEOL JEM-2010), respectively. Phase analysis was performed by powder X-ray diffraction (XRD, Bruker D8 Advance) with $\mathrm{Cu}-\mathrm{K} \alpha$ radiation $(\lambda$ $=1.540 \AA$ ) at $40 \mathrm{kV}$ with the angular increasing from $10^{\circ}$ to $70^{\circ}$. The specific surface properties of samples were studied by Brunauer-Emmett-Teller (BET, JWGB SCI\&TECH JW-BK222) methods via nitrogen adsorption and desorption measurements. Raman microscopy (Renishaw PLC) was employed to obtain the Raman spectra. X-ray photoelectron spectroscopy (XPS, ESCALAB210) analysis was employed to prove the

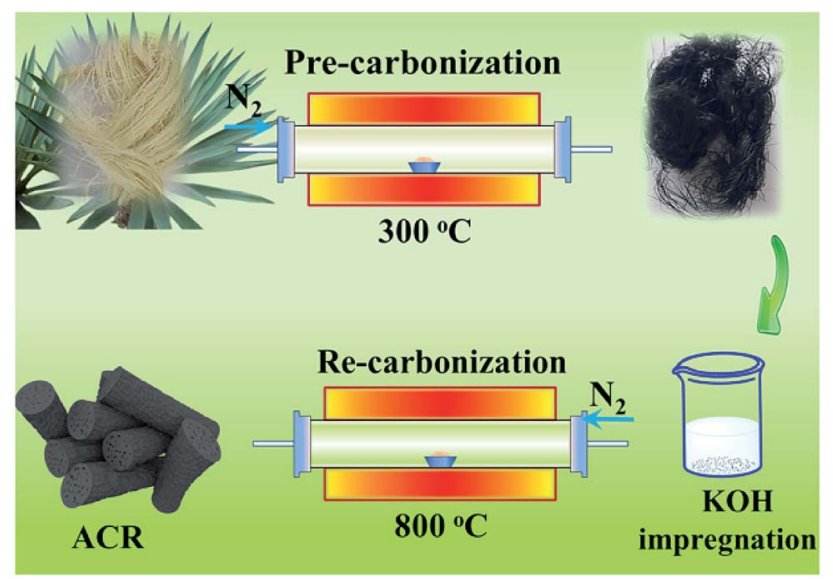

Scheme 1 Main manufacturing process of ACR. 
existence of $\mathrm{Fe}_{3} \mathrm{O}_{4}$ for FCR. Besides, to test the thermal stability, a thermogravimetric analyzer (TG, NETZSCH 209 F1) was applied, and the temperature ranged from $25{ }^{\circ} \mathrm{C}$ to $900{ }^{\circ} \mathrm{C}$ at a speed of $10{ }^{\circ} \mathrm{C} \mathrm{min}^{-1}$.

EMW absorption performances were measured by an Agilent N5224A vector network analyzer at room temperature. The asmade samples mixed by paraffin with a weight loading ratio of $30 \%$ were pressed into torus ( $\left.\Phi_{\text {out }}=7.0 \mathrm{~mm}, \Phi_{\text {in }}=3.04 \mathrm{~mm}\right)$, the complex permittivity and permeability values were measured in the $2-18 \mathrm{GHz}$ frequency range with the coaxial line method.

\section{Results and discussion}

\section{Characterization of samples}

Fig. 1 shows the SEM images of as-obtained samples CR, ACR and FCR. In visual field, undoubtedly, the major structure of all three samples is rod-like, despite a tiny bit of fragments that splitting away off the rods body after grinding. The three kinds of carbon rods that come from three different treatments all present many wrinkles and bumps on the surface, owing which the surface of rods is rough and looks like a bitter gourd. More importantly, obvious pores emerge in the cross section of CR, ACR and FCR rods. The formation of these pores mainly results from different thermal shrinkage coefficients of the three main components of sisal fiber, that is, the lignin (20-30\%), cellulose (35-50\%) and hemicellulose cellulose (20-30\%). ${ }^{20}$ According to the SEM images, for associative analysis, a thermogravimetric method was carried out to investigate the thermal stability of natural sisal fiber and the curve was shown in Fig. S1. $\dagger$ Obviously, there are three apparent turning points severally locating at 250,350 and $700{ }^{\circ} \mathrm{C}$ in the curve, suggesting that pyrolysis processes of different compositions in sisal fiber were respectively achieved under different temperature intervals. Notably,
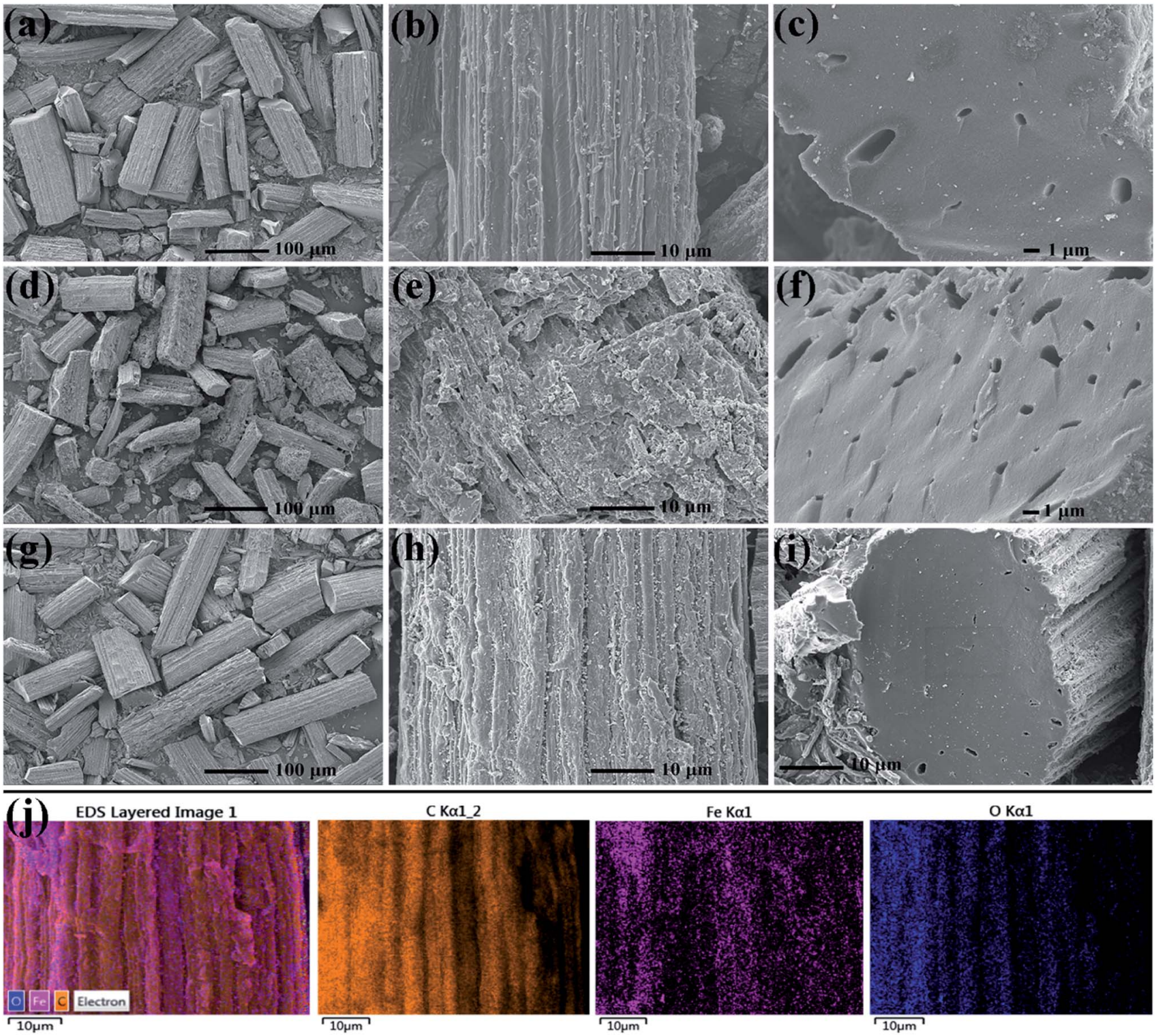

Fig. 1 SEM images of samples with different magnifications (CR ( $a-c), A C R(d-f), F C R(g-i))$, elemental mapping of FCR (j). 
after $700{ }^{\circ} \mathrm{C}$, the curve maintains flat, it reveals the end of pyrolysis and completed carbonization for fiber. Therefore, it was after the completed carbonization that the effect of thermal shrinkage in pores formation began showing and this agrees with the observation from SEM. ${ }^{21}$ The rough surface provided by wrinkles and bumps could increase the interface between carbon rods and air, thereby lead to improved interfacial polarization and more EMW would enter the absorber to be attenuated..$^{22,23}$ Similarly, the voids came from the micron-scale macropores also offer additional interface, and besides, multireflection could happen in these pores. ${ }^{\mathbf{2 4 , 2 5}}$ Hence, the special microstructure of these carbon rods helps to benefit EMW absorption.

Further details of micro-morphology and structure are shown in Fig. 2 of representative TEM images. It is noteworthy that many black spots on the rod are exhibited in the projection of Fig. 2a and c for CR and ACR sample, the black spots are considered as inorganic salt crystals derived from the innate trace amount of metal ions like $\mathrm{K}^{+}, \mathrm{Ca}^{2+}, \mathrm{Na}^{+}, \mathrm{Fe}^{2+} / \mathrm{Fe}^{3+}$ or any others in natural sisal fiber. In contrast, the PCR TEM image in Fig. $\mathrm{S} 2 \dagger$ is smooth and clean, even almost no black spots are observed, illustrating that the trace inorganic salt ions in PCR were removed after acid pickling. These inherent ions served as doped heteroatoms could play the part of polarization centers and help to attenuate EMW. ${ }^{26}$ Accordingly, there should be some lifted difference between CR and PCR, and complete details of this case in EMW absorption performance will be provided at below. Compared with CR and ACR, FCR shows dense black spots in Fig. 2e, the spots density of FCR is obviously higher than that of CR and ACR. Most of these black spots are believed to be the generated $\mathrm{Fe}_{3} \mathrm{O}_{4}$ particles, and it is confirmed in Fig. $2 \mathrm{~g}$. In the high resolution image of Fig. $2 \mathrm{~g}$, these lattice fringes coincide well with the (311) plane of $\mathrm{Fe}_{3} \mathrm{O}_{4}$, indicating that these particles are $\mathrm{Fe}_{3} \mathrm{O}_{4}$ crystals. Magnetic $\mathrm{Fe}_{3} \mathrm{O}_{4}$ particles were formed by iron salt impregnation in the subsequent carbonization of FCR. Supplementary saturation magnetization curve of FCR is provided in Fig. $\mathrm{S} 3, \uparrow$ it can be seen that FCR has good paramagnetism. Additionally, the XPS

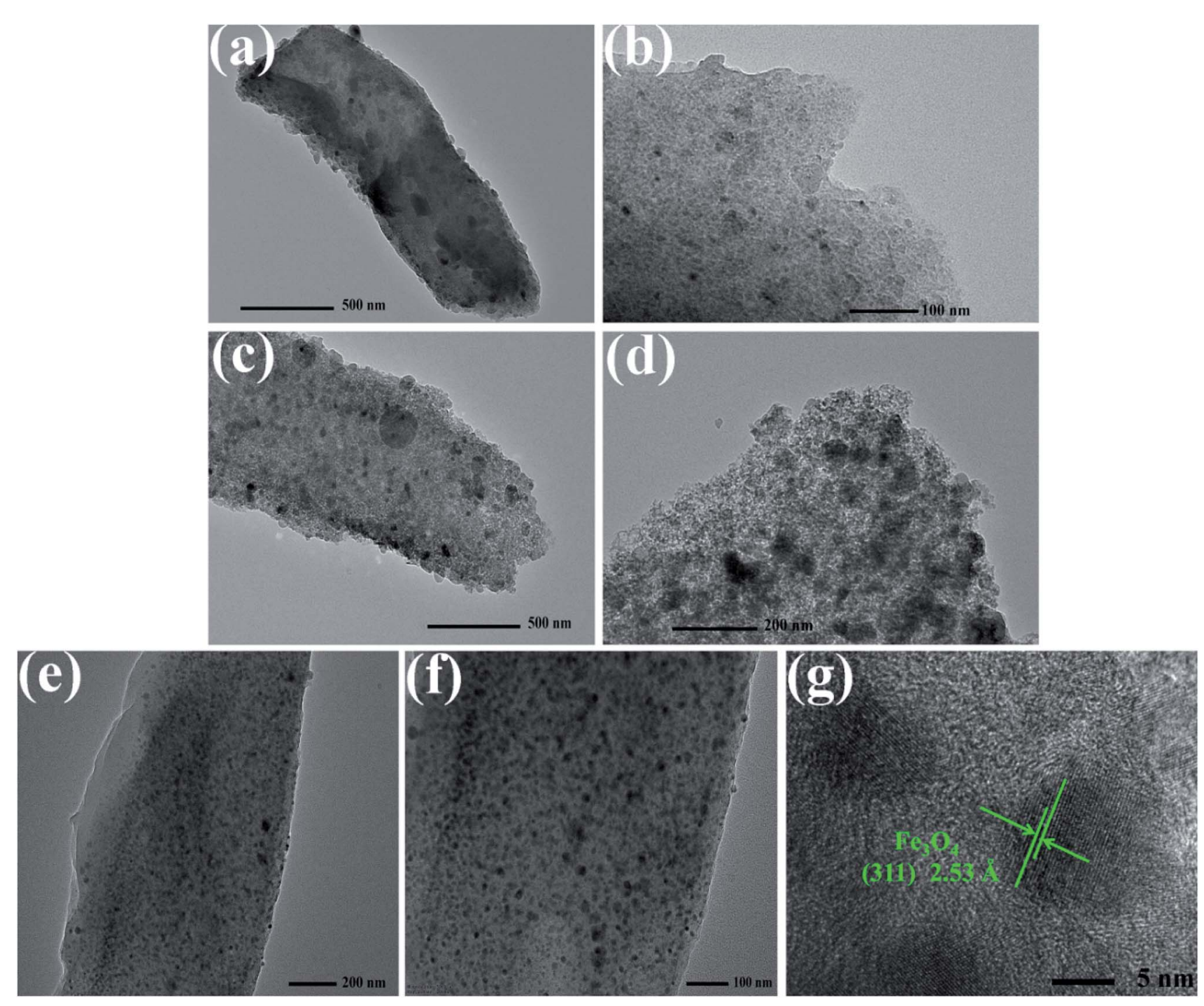

Fig. 2 TEM images of samples (CR ( $a$ and b), ACR ( $c$ and d) and FCR (e and g)) with different magnifications. 
spectra in Fig. $\mathrm{S} 4 \dagger$ were also delivered to describe the Fe element state in FCR. It can be found in Fig. $\mathrm{S} 4 \mathrm{~b} \dagger$ that the Fe $2 \mathrm{p}$ peaks are mainly centered at 707.90 and $721.40 \mathrm{eV}$, corresponding to $\mathrm{Fe} 2 \mathrm{p} 1 / 2$ and $\mathrm{Fe} 2 \mathrm{p} 3 / 2$ state in $\mathrm{Fe}_{3} \mathrm{O}_{4}$. Besides, another small satellite peak at $715.20 \mathrm{eV}$ was aroused by Fe 2p3/ 2 state in trace amount of elemental iron.

Fig. 3 presents the XRD patterns, Raman spectra and $\mathrm{N}_{2}$ adsorption-desorption isotherms of CR, ACR and FCR. In Fig. 3a, ACR and CR characterized two broad peaks that locating at $\sim 25^{\circ}$ and $42^{\circ}$, assigning to the classical (002) and (100) crystal plane of hexagonal graphite (ICSD \#85-1436), and no other obvious impurity peaks detected in the patterns. The trace metal elements did not give recognizable diffraction peaks might because of the low content, or weaker diffraction peaks were concealed by the stronger peaks from hexagonal graphite. For FCR, beyond the graphite peaks, the sharp and obvious diffraction peaks correspond well with the standard peaks of face center cubic spinel phase $\mathrm{Fe}_{3} \mathrm{O}_{4}$ (ICSD \#85-1436), which is consistent with previous TEM analysis.

Moreover, Raman spectra were used to evaluate the graphitization degree of samples. In general, two distinguishable peaks standing at 1340 and $1590 \mathrm{~cm}^{-1}$ are named as D band $\left(\mathrm{A}_{1 \mathrm{~g}}\right.$ carbon vibration modes) and $\mathrm{G}$ band ( $\mathrm{E}_{2 \mathrm{~g}}$ carbon vibration modes). ${ }^{27,28}$ The $\mathrm{D}$ band usually associates with disorder carbon which contains vacancies, substitutional heteroatoms, amorphous carbon species, grain boundaries or any other defects, while the $\mathrm{G}$ band is subject to $\mathrm{sp}^{2}$-hybridized carbon bonds, which can be produced by all $\mathrm{sp}^{2}$ sites in graphitic carbon. ${ }^{29-31}$ The integrated intensity ratio of $\mathrm{D}$ band and $\mathrm{G}$ band $\left(I_{\mathrm{D}} / I_{\mathrm{G}}\right)$, acquired from an accurate calculation by integral peak area, is usually employed as a criterion to reflect the graphitization degree of sample. ${ }^{32}$ In Fig. 3b, the as-prepared sample ACR, CR and FCR all present a high $I_{\mathrm{D}} / I_{\mathrm{G}}$ value that is greater than 1.5 , and the corresponding values are $1.94,1.76$, and 1.83 , respectively. In contrast to other published works which focus on chemically synthesized carbonaceous materials in this field, ${ }^{33-37}$ the carbon rods derived from sisal fiber give rare and noticeable high $I_{\mathrm{D}} / I_{\mathrm{G}}$ value due to low graphitization degree, in other words, plenty of defects exist in the amorphous/disorder carbon. The trace metal ions in sisal fiber, serving as doped heteroatoms, are much likely to cause the lattice distortions of surrounding carbon and facilitate the generation of various defects such as vacancies and misshapen grain boundaries or any others during calcination. ${ }^{\mathbf{1 4 , 1 6}}$ In addition, different carbon sources (lignin, cellulose and hemicellulose cellulose), as well as different micro organizational structures in biomass (cell wall, vascular bundle, conduit, etc.), could also form different interfaces in carbonaceous matrix during pyrolysis, and many defects would generate on these heterogeneous interfaces. In addition to the defects caused by these inherent factors, $\mathrm{KOH}$ etching activation or $\mathrm{Fe}_{3} \mathrm{O}_{4}$ particles loading further help to improve the defect level of ACR or FCR, respectively. Normally,
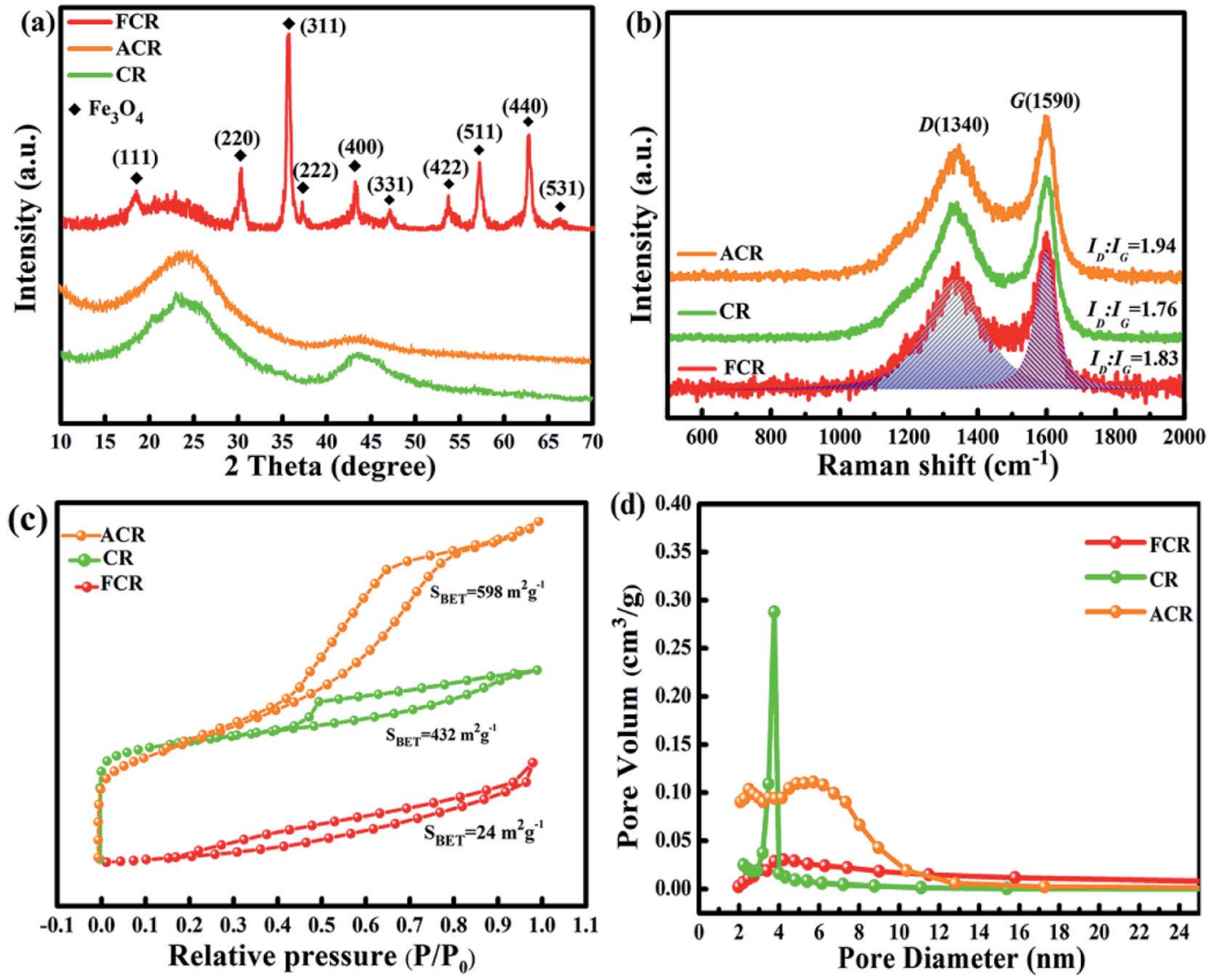

Fig. 3 XRD patterns (a), Raman spectra (b), $\mathrm{N}_{2}$ adsorption-desorption isotherms (c) and pore size distribution plots (d) of FCR, ACR and CR. 

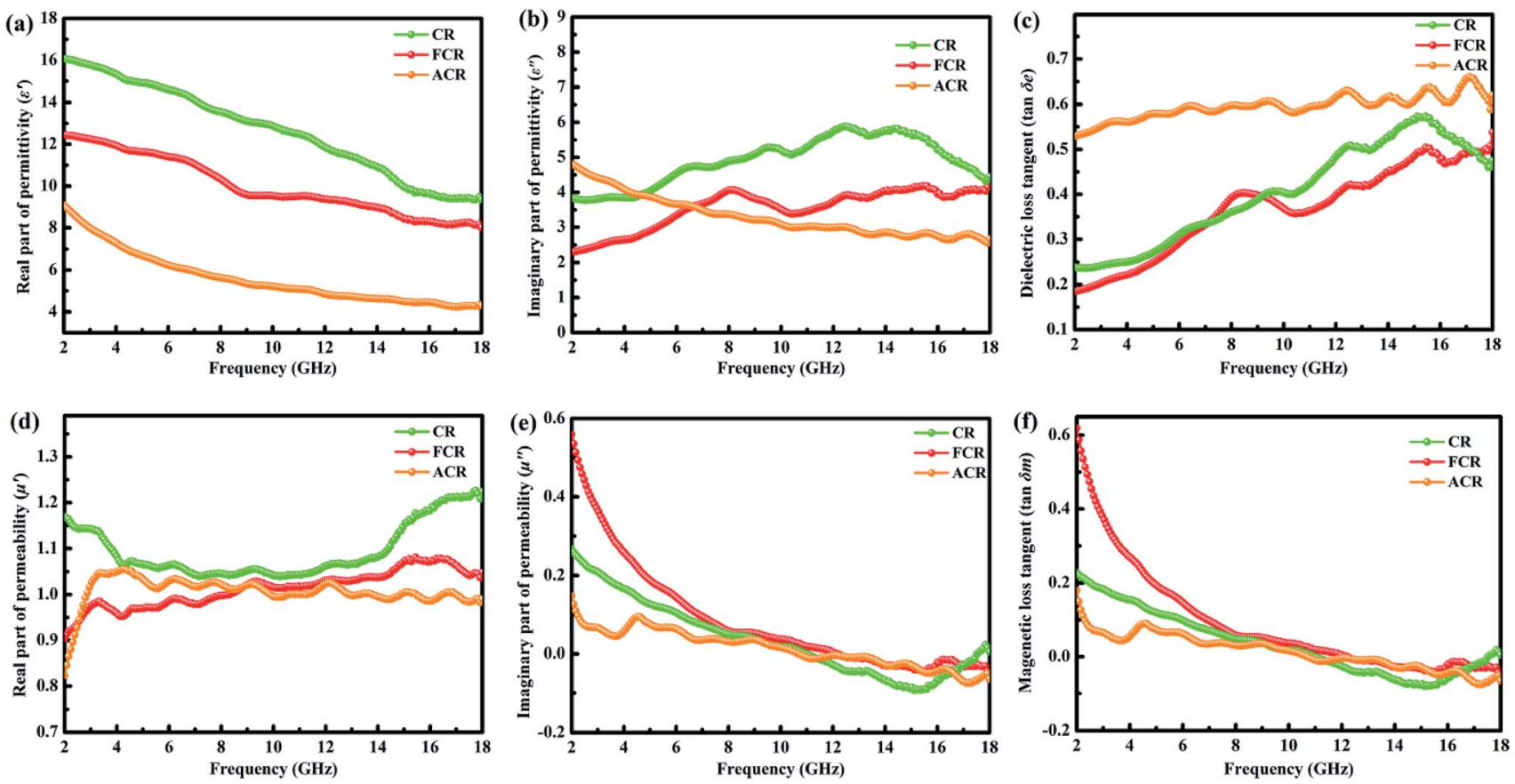

Fig. 4 Frequency dependence of the real part (a) and imaginary part (b) of permittivity, the real part (d) and imaginary part (e) of permeability, and the dielectric (c) and magnetic (f) tangent loss values of three samples.

the formed defects not only introduce defect polarization relaxation and dipole polarization relaxation, but also promote the transition from contiguous state to Fermi level, all of these are in favor of EMW penetration and absorption. ${ }^{38,39}$

$\mathrm{N}_{2}$ adsorption-desorption isotherms were measured to study the surface area, pore size of samples. Fig. 3c plots the typical type IV isotherms with large hysteresis loops for ACR and CR, which indicates the characteristic of mesoporous materials according to the IUPAC classification.$^{40}$ By comparison, FCR protrudes downward throughout the pressure range, and presents type III isotherm. Fig. 3d presents pore size distribution plots of three samples. Three obtained samples have little difference in pore diameter, all show the characteristics of micropores. As mentioned in SEM analysis, the carbon rods cross section shows the macropores, but the macropores have little contribution to the specific surface area $\left(S_{\mathrm{BET}}\right)$ of material, instead, the micropores actually enlarge the $S_{\mathrm{BET}}$. The two-level porous structure could improve interface polarization, then greatly perfect the behavior of EMW absorption..$^{41}$ Activated by $\mathrm{KOH}$, the $S_{\mathrm{BET}}$ of ACR increased by nearly 170 compared with CR, while the $S_{\text {BET }}$ of FCR with loaded $\mathrm{Fe}_{3} \mathrm{O}_{4}$ particles is very low. It is due to the large number of $\mathrm{Fe}_{3} \mathrm{O}_{4}$ particles which greatly increase the unit mass of FCR.

\section{EMM absorption properties of samples}

The two decisive parameters, relative complex permittivity $\left(\varepsilon_{\mathrm{r}}=\right.$ $\left.\varepsilon^{\prime}-j \varepsilon^{\prime \prime}\right)$ and relative complex permeability $\left(\mu_{\mathrm{r}}=\mu^{\prime}-j \mu^{\prime \prime}\right)$ of samples, were measured with the frequency ranging from 2 to $18 \mathrm{GHz}$ to study the EMW absorptivity. Ordinarily, the real parts of the complex permittivity $\left(\varepsilon^{\prime}\right)$ and permeability $\left(\mu^{\prime}\right)$ represents the storage capability of electric and magnetic energy, while the imaginary parts $\left(\varepsilon^{\prime \prime}\right.$ and $\left.\mu^{\prime \prime}\right)$ are on behalf of the dissipation capability of electric and magnetic energy. ${ }^{42,43}$ The $\varepsilon^{\prime}, \varepsilon^{\prime \prime}, \mu^{\prime}$ and $\mu^{\prime \prime}$ depending on frequency are depicted in Fig. 4 .

As can be seen clearly in Fig. 4a and b, intuitive differences exist between three samples, especially the CR, which plots the highest $\varepsilon^{\prime}$ and $\varepsilon^{\prime \prime}$ values over the most tested frequency range. In Fig. $4 \mathrm{~b}$ of $\varepsilon^{\prime \prime}$ versus frequency, all three samples exhibit obvious resonance peaks at high frequencies. The multiple resonance peaks of $\varepsilon^{\prime \prime}$ are caused by various polarization relaxations, e.g. interfacial polarization, electronic polarization and atomic polarization (of course, here, interface polarization plays a major role). ${ }^{13,27,32,44}$ This kind of relaxation is due to the fact that polarization cannot keep up with the rapidly changing of alternate electric field in high frequency region. Usually, the higher the frequency is, the more distinct the relaxation phenomenon is, account for this, resonance relaxation peak occurs in the high frequency region. Those multiple resonances reflected in peaks ascribe to the special microstructure, namely, innate trace metal ions and defects, as well as interfaces between solid-void, air-surface roughness and heterojunctions in carbon rods, all are believed to supply active sites for polarization. ${ }^{34,42}$ According to Debye theory, polarization relaxation could be considered as one of the key factors to positively contribute to the complex permittivity and then benefit the EMW absorption. ${ }^{4,45}$ Furthermore, as mentioned in Yin's classical review article, only with appropriate $\varepsilon^{\prime}$ and $\varepsilon^{\prime \prime}$ values can material have EMW absorption, that is, low $\varepsilon^{\prime}$ and intermediate $\varepsilon^{\prime \prime}$ are basic requirements, the author also pointed out the required dielectric properties of EMW absorption materials for application, and the specific values region is also given out: $\varepsilon^{\prime}$ ranges from 5 to $20, \varepsilon^{\prime \prime}$ ranges from 1 to $10 .^{46}$ Fortunately, our 

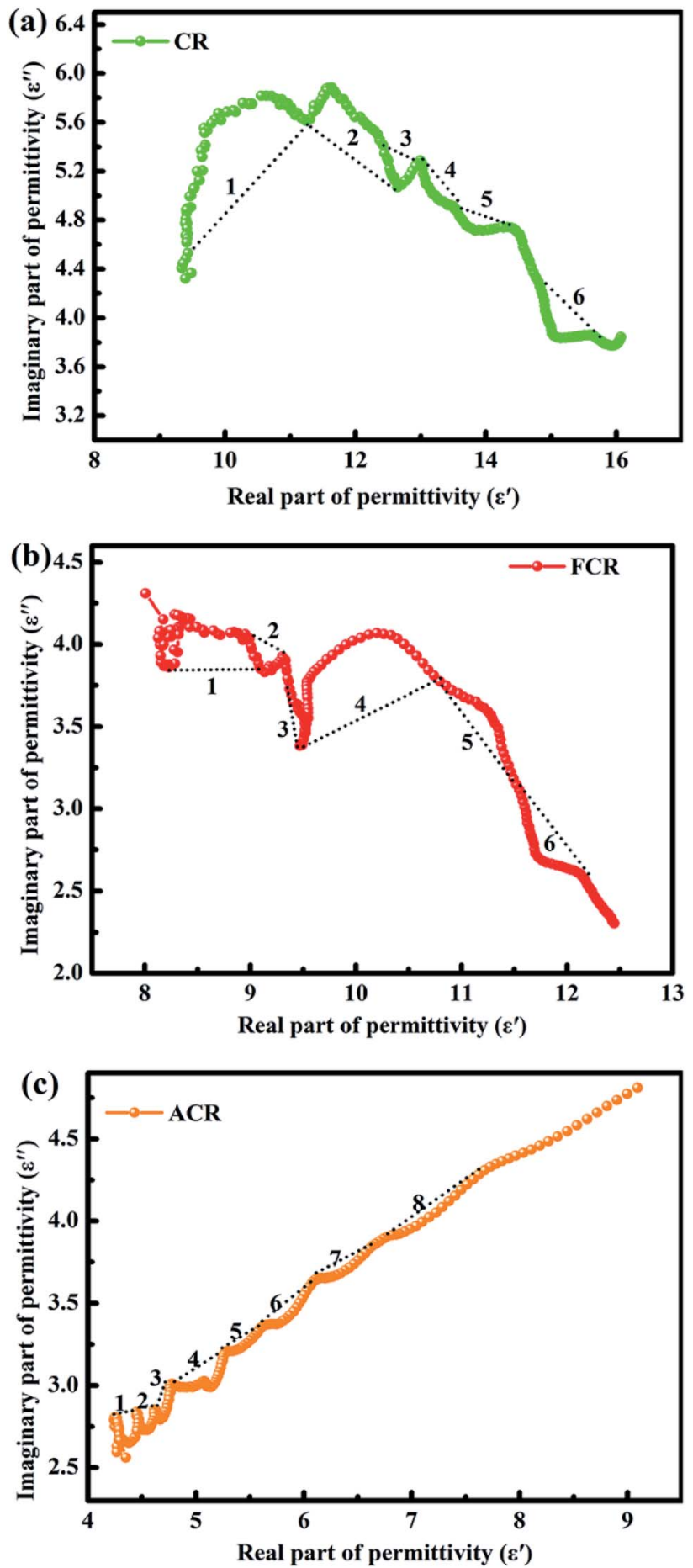

Fig. 5 Cole-Cole plots of CR, FCR and ACR.

materials just meet this requirement. The dielectric loss tangents $\left(\tan \delta_{\mathrm{e}}=\varepsilon^{\prime \prime} / \varepsilon^{\prime}\right.$, Fig. 4c) were calculated to evaluate the dielectric loss ability. ${ }^{47}$ Apparently, ACR gives the highest tangent value upon all frequency. ACR presents higher $S_{\mathrm{BET}}$ and $I_{\mathrm{D}} / I_{\mathrm{G}}$ values, which mean large scale mesopores and defects staying in it. On the one hand, abundant pores could provide enough additional solid-void interfaces, followed by which a considerable amount of interface polarization occurs. ${ }^{48,49} \mathrm{On}$
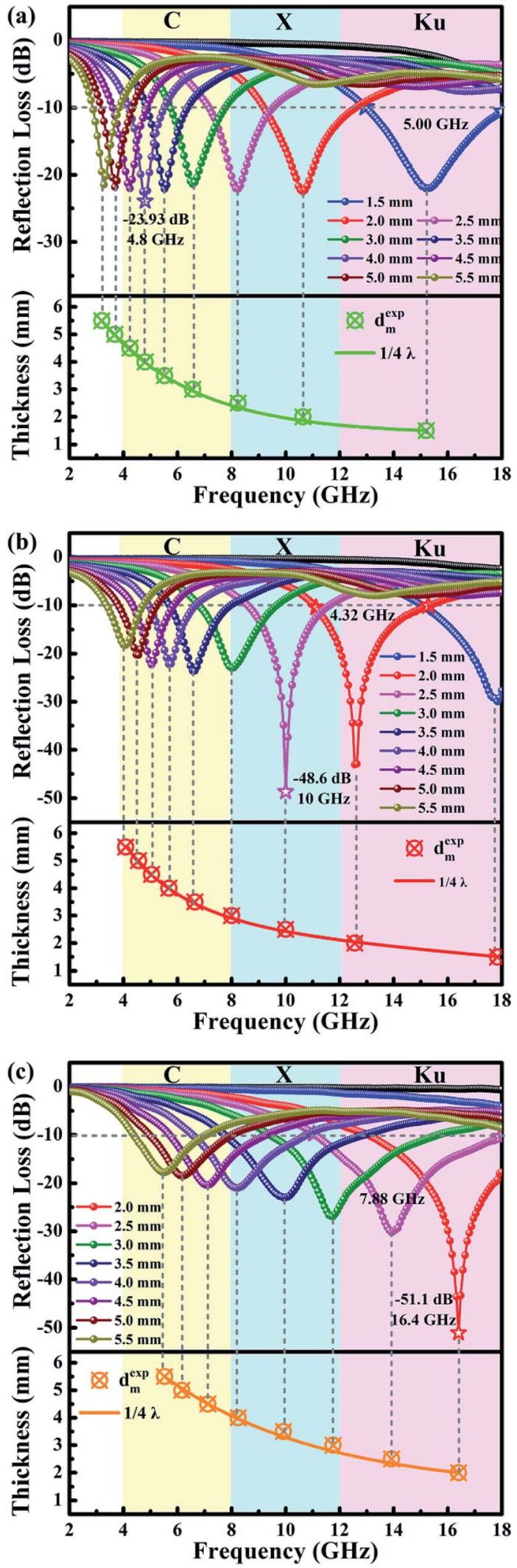

Fig. 6 RL curves of $C R(a), F C R(b)$ and ACR (c). The bottom of RL curves is emulation of the peak frequency $\left(f_{m}\right)$ vs. absorber thickness $\left(d_{m}\right)$ under $\lambda / 4$ model. 
the other hand, plentiful defects serve as polarization centers to arouse defect dipolar polarization. ${ }^{22,50}$ These two polarizations would induce dense charges accumulating in the presence of EMW, subsequently, the conductivity improves and the dielectric loss polishes up according to free electron theory. ${ }^{\mathbf{1 4 , 5 1 , 5 2}}$

For most EMW absorbers, particularly the synthetic composites, ferromagnetic components are often used to modify the materials and improve the magnetic loss. ${ }^{53,54}$ Here, another modification of our carbon rods is magnetic modification. In Fig. 4d, CR exhibits the highest $\mu^{\prime}$ value over the whole frequency range, revealing $\mathrm{CR}$ has the best electromagnetic energy storage capacity. Whereas in $\mu^{\prime \prime}$ plots of Fig. 4e, magnetic FCR is almost the leader of three samples over all tested frequency range, indicating that FCR occupies superior dissipative ability of electromagnetic energy. Besides, all the three samples show negative values at high frequency regio, manifesting that magnetic energy was partially radiated out from the samples. ${ }^{49,55}$ The magnetic loss tangents $\left(\tan \delta_{\mathrm{m}}=\mu^{\prime \prime}\right.$ / $\mu^{\prime}$, Fig. 4f) display that the magnetic loss of FCR is obviously superior to that of the other two samples by loading magnetic particles.

To further understand the significance of dielectric properties in improving material absorbing behaviors, Cole-Cole plots deduced by $\varepsilon^{\prime} v s$. $\varepsilon^{\prime \prime}$ from Debye polarization relaxation theory were carried out, the equation is shown below: ${ }^{56,57}$

$$
\left(\varepsilon^{\prime}-\frac{\varepsilon_{\mathrm{s}}-\varepsilon_{\infty}}{2}\right)^{2}+\left(\varepsilon^{\prime \prime}\right)^{2}=\left(\frac{\varepsilon_{\mathrm{s}}-\varepsilon_{\infty}}{2}\right)^{2}
$$

Here, $\varepsilon_{\mathrm{S}}$ is the static dielectric constant and $\varepsilon_{\infty}$ corresponds the dielectric constant at infinite frequency. ${ }^{58}$ According to the equation, Cole-Cole plots should be shown as a semicircle, and a Cole-Cole semicircle represents a Debye relaxation process. ${ }^{59}$ In Fig. 5, it can be clearly seen that ACR has much overlapping and distorted semicircles than that of CR and FCR, in other words, CR and FCR has fewer relaxation processes when in contrast with the activated ACR, this result agrees well with the foregoing interpretation of $\varepsilon^{\prime}$ and $\varepsilon^{\prime \prime}$.

The most intuitive date of EMW absorbency is reflection loss $(\mathrm{RL})$, which is calculated from the transmission line theory: ${ }^{\mathbf{6 0 , 6 1}}$

$$
\begin{aligned}
& Z_{\text {in }}=Z_{0}\left(\mu_{\mathrm{r}} / \varepsilon_{\mathrm{r}}\right)^{1 / 2} \tanh \left[j(2 \pi f d / c)\left(\mu_{\mathrm{r}} \varepsilon_{\mathrm{r}}\right)^{1 / 2}\right] \\
& \mathrm{RL}(\mathrm{dB})=20 \log \left|\left(Z_{\text {in }}-Z_{0}\right) /\left(Z_{\text {in }}+Z_{0}\right)\right|
\end{aligned}
$$

where, $Z_{\text {in }}$ is the input characteristic impedance, while $Z_{0}$ presents the impedance of free space, $c$ corresponds to the velocity of light, $f$ is the frequency and $d$ is the thickness of the absorber, respectively. ${ }^{62}$ In practical applications, the RL value under $-10 \mathrm{~dB}$ means that more than $90 \%$ of the incident EMW be absorbed, correspondingly, when the RL is lower than -20 or $-30 \mathrm{~dB}$, manifesting 99 or $99.9 \%$ of the EMW is absorbed. ${ }^{63}$ Fig. 6 displays the EMW absorption performance of prepared samples in an all-round way by plane illustrations. CR that without any modification in Fig. 6a show mediocre EMW absorbing performance, of which the minimum RL is -23.93 $\mathrm{dB}$ at $4.8 \mathrm{GHz}$ with the thickness of $4.0 \mathrm{~mm}$, and the maximum absorbing bandwidth (MAB) under $-10 \mathrm{~dB}$ is $5.0 \mathrm{GHz}$ from
13.00 to $18.00 \mathrm{GHz}$. Moreover, the RL values of CR under all tested thicknesses are below $-20 \mathrm{~dB}$, indicating that the intrinsic structure come from sisal fiber without any modification is beneficial to the absorption of EMW. Besides, the absorbing frequency completely covers $\mathrm{C}, \mathrm{X}$ and $\mathrm{Ku}$ band when adjusting the thickness from 1.5 to $4.5 \mathrm{~mm}$. A simple comparison between CR and PCR were carried out to investigate the influence of inherent trace metal ions of carbon rods on EMW absorption. As mentioned earlier, the internal metal ions in PCR have been removed by acid pickling. Fig. S5† plots the RL details of PCR. Even though PCR presents strong absorption, the corresponding absorbing thickness is relatively thicker, which is far from being comparable to CR absorbing performance. It exposes a fact: PCR which experienced a pickling process after carbonization lost the embedded trace metal ions, afterwards performed poor absorbency. By this, the positive effect of intrinsic inorganic metal ions in EMW absorbing was emphasized. As for FCR, in Fig. 6b, it manifests beautiful RL curves with different absorbing thickness, the minimum RL reaches $-48.6 \mathrm{~dB}$ at $10 \mathrm{GHz}$ with a thickness of $2.5 \mathrm{~mm}$, the MAB is $4.32 \mathrm{GHz}$ in $2.0 \mathrm{~mm}$ when the frequency ranges from 11.02 to $15.34 \mathrm{GHz}$. In contrast with unmodified CR, FCR loaded with $\mathrm{Fe}_{3} \mathrm{O}_{4}$ particles improved its magnetic loss after magnetic modification on the basis of original characteristics of sisal fiber, thereby an enhanced EMW absorption coming from the synergistic effect of dielectric loss and magnetic loss appears. ACR, which activated by $\mathrm{KOH}$, possess the top absorbing capacity among three tested samples and the RL curves is depicted in Fig. 6c. The minimum RL of ACR achieves $-51.1 \mathrm{~dB}$ with the thickness of $2.0 \mathrm{~mm}$ at $16.4 \mathrm{GHz}$, while the MAB achieves $7.88 \mathrm{GHz}$ from 11.12 to $18.00 \mathrm{GHz}$ of $2.5 \mathrm{~mm}$, which completely cover the $\mathrm{Ku}$ band via a single thickness. Compared with sample FCR, ACR possessing non-magnetic constituent presents stronger absorption ability, it is mainly attributed to the change of microstructure and the improvement of dielectric loss after $\mathrm{KOH}$ etching. Through the etching activation, the porosity and defect levels of ACR are higher than those of FCR, and then the dielectric loss is greatly increased. Forceful dielectric loss further promotes strong absorption performance, thus the sample ACR stands out.

Crucial like frequency, the thickness of absorber play an equally important role to determine the practical application. In addition to the strong absorption from the absorber itself, partial incident EMW can also be attenuated by a "geometric effect", which is called the quarter wavelength $(\lambda / 4)$ model theory. ${ }^{31,64}$ From the equation: ${ }^{65}$

$$
d_{\mathrm{m}}=n \lambda / 4=n c /\left[4 f_{\mathrm{m}} \sqrt{ }\left(\left|\varepsilon_{\mathrm{r}} \| \mu_{\mathrm{r}}\right|\right)\right](n=1,3,5, \ldots)
$$

Herein, $d_{\mathrm{m}}$ represents the absorber thickness, $\lambda$ is the EMW wavelength and $f_{\mathrm{m}}$ corresponds the frequency of the minimum RL positions. According to the $\lambda / 4$ model theory, when $d_{\mathrm{m}}$ and $f_{\mathrm{m}}$ meet the equation, a phase difference which is right $180^{\circ}$ between incident and reflected EMW would occur on the airabsorber interface, subsequently, the special interference loss, or named cancellation effect, is induced and leading to extinction of these specific EMW on the interface. ${ }^{\mathbf{1 2 , 6 6}}$ The bottom of 

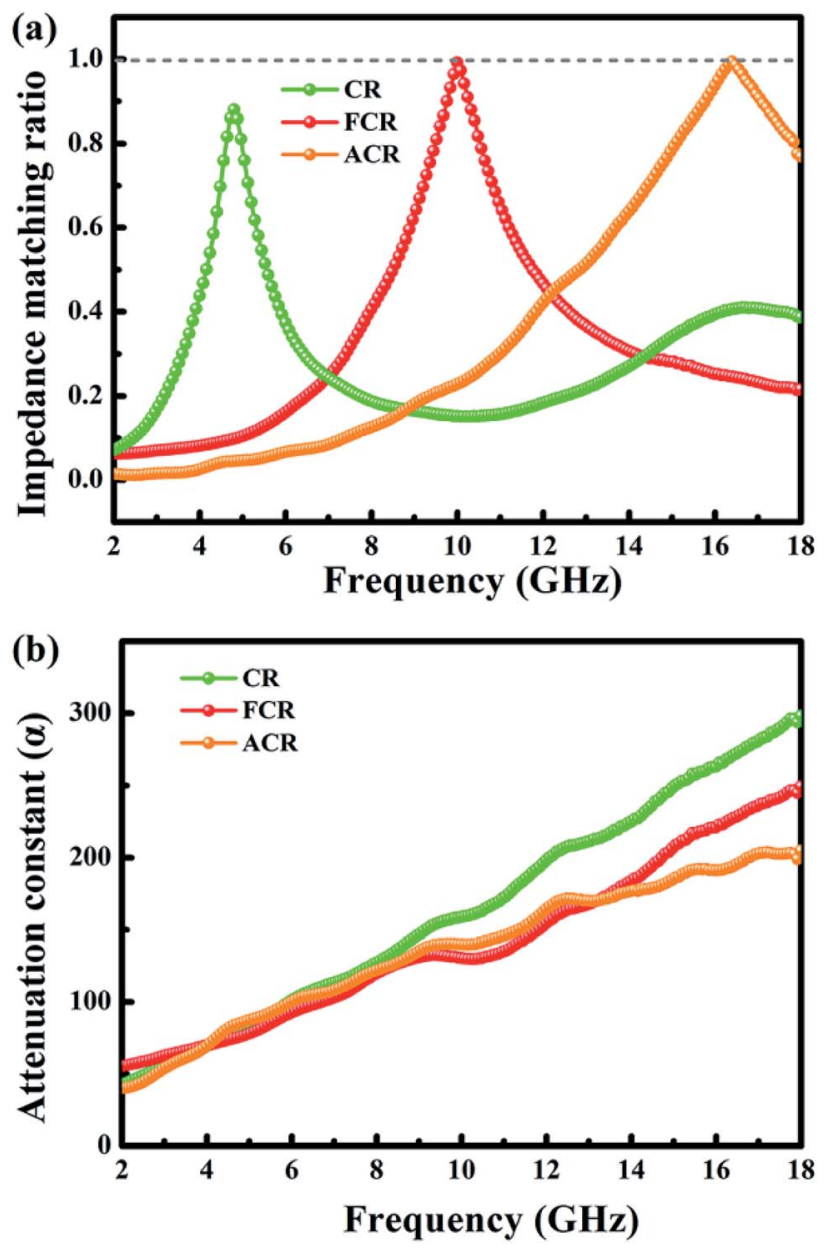

Fig. 7 The normalized impendence matching ratio (a) and attenuation constant (b) of three samples.

Fig. 6a-c plot the frequency of minimum RL $\left(f_{\mathrm{m}}\right) v s$. absorber thickness $\left(d_{\mathrm{m}}\right)$ for CR, FCR and ACR. The cycles denoted as $d_{\mathrm{m}}^{\exp }$ are the experimental thicknesses and the smooth curve is emulated from $\lambda / 4$ model. Clearly, all the $d_{\mathrm{m}}^{\exp }$ exactly fall in the $\lambda / 4$ curve, signifies that absorption thickness and frequency of each sample entirely match the $\lambda / 4$ model and interference loss happened in them. Moreover, the $\lambda / 4$ theory also plays a referential guidance to design absorption materials with appropriate thickness once required electromagnetic properties are obtained.

The normalized impendence matching ratio $\left(Z=\left|Z_{\text {in }} / Z_{0}\right|\right)$ and attenuation constant $(\alpha)$ were calculated and provided extra evidences to expound the contrast differences of ultimate EMW absorption ability between three samples. The impendence matching will be better when $Z$ is much closer to 1 , under this circumstance, the outside incident EMW enters into absorber as much as possible and then to be further attenuated. ${ }^{67}$ Fig. 7a renders the optimal impendence matching ratio with frequency variation for three samples. ACR and FCR show expected $Z$ value of 1 . However, the $Z$ value of CR is lower than 1 , suggesting that a considerable number of incident EMW is reflected back from the surface of absorber, instead of entering into the absorber interior. Therefore, the absorption of EMW by CR is not as good as the other two.

Attenuation constant $(\alpha)$ was introduced to quantificate the ability of absorber attenuating interior EMW, the value of $\alpha$ can be calculated by means of the following equation: $:^{30,45}$

$$
\begin{aligned}
\alpha=(\sqrt{ } 2 \pi f / c) & \times \sqrt{ }\left[\left(\mu^{\prime \prime} \varepsilon^{\prime \prime}-\mu^{\prime} \varepsilon^{\prime}\right)+\sqrt{ }\left[\left(\mu^{\prime \prime} \varepsilon^{\prime \prime}-\mu^{\prime} \varepsilon^{\prime}\right)^{2}\right.\right. \\
& \left.\left.+\left(\mu^{\prime \prime} \varepsilon^{\prime \prime}+\mu^{\prime} \varepsilon^{\prime}\right)^{2}\right]\right]
\end{aligned}
$$

In Fig. 7b, the relying of attenuation constant $\alpha$ on frequency presents that CR exhibits the highest $\alpha$ value in most frequency region, while ACR and FCR are slightly inferior. Nevertheless, because the impedance matching of CR is the lowest, that is, the amount of EMW that can enter its interior is the least, thus the overall absorption effect of CR to EMW is not satisfied. Impedance matching is a prerequisite for EMW absorbing materials, and it determines whether the external EMW can enter the absorber rather than being reflected back.

Related comparative works derived from biomass have been listed out in Table 1. Biomass-derived materials which stem from sisal fiber (this work), chicken feather, spinach stem or any others perform excellent EMW absorbing behaviors, and in a comprehensive way, our carbon rods bear favourable technical comparison by strong RL and broad MAB. Furthermore, the two modification methods for materials here are commonly used and easy to carry out, and help to achieved ideal results. To sum up, all the carbonaceous absorbers listed in Table 1 could be used as a referable paradigmatic for making full use of biomass materials in this fascinating field, and with integrative

\begin{tabular}{|c|c|c|c|c|c|c|}
\hline Absorber & Raw materials & Content (wt\%) & $\mathrm{RL}_{\min }(\mathrm{dB})$ & $\begin{array}{l}\text { Thickness } \\
(\mathrm{mm})\end{array}$ & $\mathrm{MAB}(\mathrm{GHz})$ & Ref. \\
\hline Activated carbon rods & Sisal fiber & 30 & -51.1 & 2.0 & 7.88 & Herein \\
\hline Magnetic carbon rods & Sisal fiber & 30 & -48.6 & 2.5 & 4.32 & Herein \\
\hline Porous carbon & Spinach stem & 30 & -41.2 & 1.5 & 4.60 & 14 \\
\hline Heteroatom-doped carbon & Chicken feather & 30 & -20.1 & 2.0 & 2.90 & 16 \\
\hline Porous magnetic carbon & Rice & 15 & -52 & 1.7 & 5.0 & 15 \\
\hline Porous carbon & Walnut shell & - & -42.4 & 2.0 & 2.24 & 50 \\
\hline Ferromagnetic carbon nanofiber & Collagen fiber & 38.1 & -36 & 2.0 & 5.40 & 17 \\
\hline $\mathrm{Ni}(\mathrm{OH})_{2} /$ carbon & Jackfruit peel & 50 & -23.6 & 6.0 & - & 68 \\
\hline Carbon-cotton/Co@nanoporous carbon & Cotton & 25 & -60 & 2.55 & 4.40 & 69 \\
\hline
\end{tabular}

Table 1 Comparative studies on EMW absorbers prepared form biomass materials 


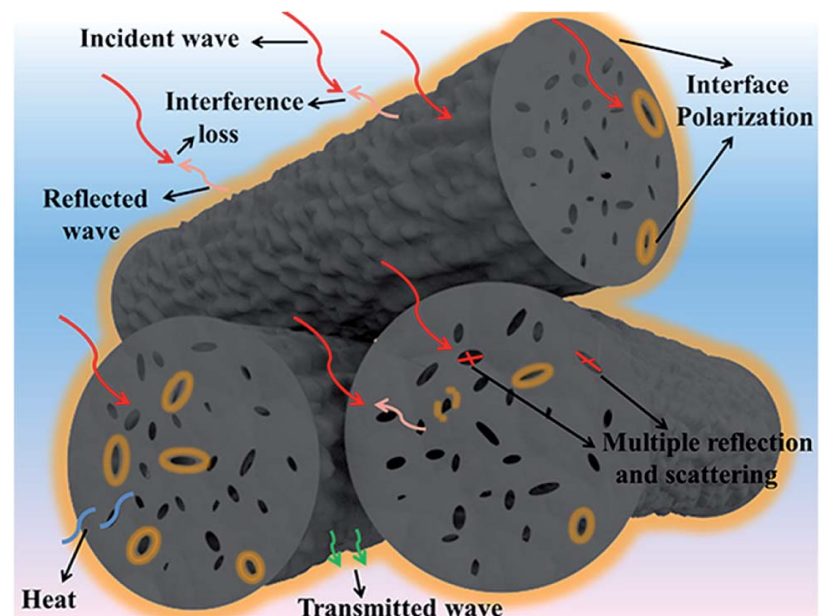

Scheme 2 Probable schematic illustration for EMW absorption of representative ACR.

consideration, it is reasonable for these carbon rods to be talent showing itself between them because of the ubiquitous material and high efficiency.

Given the abovementioned investigation and analysis, it can be rationally concluded that the optimal EMW absorbency of these representative porous carbon rods mainly originate from the beneficial structure and proper dielectric loss or magnetic loss. For one thing, numerous co-existing macropores attenuate EMW to a large extent by multiple reflection and scattering, for another, the hetero-interfaces, such as rough surface-air, voidair, carbon matrix-air, carbon matrix-trace metal and so on, greatly promote interfacial polarization and relaxation, followed by which the forceful dielectric loss emerges and consumes EMW. Specially, the magnetic loss aroused by loading magnetic $\mathrm{Fe}_{3} \mathrm{O}_{4}$ consumes EMW for FCR in another way. Aside from these, interference loss also makes contribution to the cancellation of incident wave. Probable schematic illustration for EMW absorption of representative ACR appears in Scheme 2.

\section{Conclusion}

We have shown for the first time the carbon rods derived from plant sisal fiber with eye-catching performance in electromagnetic wave absorption. The starting material is ubiquitous, environmentally friendly, and low-cost. Based on bio-inspired microstructure, the sisal fiber-derived product modified by $\mathrm{KOH}$ etching or magnetic particles loading performed excellent EMW absorbing properties, both the final reflection loss and the absorption bandwidth are satisfactory. Compared with other works which consume costly materials and complex fabrication process, our approach of the EMW absorber shows interesting and unique advantages, and conforms to current green and sustainable research trend. As thereby, it can be firmly believed that these carbon rods could be an admirable candidate in EMW absorbing, and even could also provide a feasible reference for others who want to do similar works.

\section{Conflicts of interest}

The authors declare that they have no conflicts of interest.

\section{Acknowledgements}

This project was financially supported by the National Key R\&D Program of China (2017YFB0308701), the National Natural Science Foundation of China (21676039), State Key Laboratory of Bio-Fibers and Eco-Textiles (2017kfkt12) and Innovative Talents in Liaoning Universities and Colleges (LR2017045) are highly appreciated.

\section{References}

1 B. Zhao, X. Q. Guo, W. Y. Zhao, J. S. Deng, B. Fan, G. Shao, Z. Y. Bai and R. Zhang, Nano Res., 2016, 10, 331-343.

2 C. Luo, T. Jiao, Y. Tang and J. Kong, Adv. Eng. Mater., 2018, 20, 1701168.

3 C. Luo, T. Jiao, J. Gu, Y. Tang and J. Kong, ACS Appl. Mater. Interfaces, 2018, 10, 39307-39318.

4 M. Lu, W. Cao, H. Shi, X. Fang, J. Yang, Z. Hou, H. Jin, W. Wang, J. Yuan and M. Cao, J. Mater. Chem. A, 2014, 2, 10540-10547.

5 C. Luo, Y. Tang, T. Jiao and J. Kong, ACS Appl. Mater. Interfaces, 2018, 10, 28051-28061.

6 B. Zhao, X. Zhang, J. Deng, Z. Bai, L. Liang, Y. Li and R. Zhang, Phys. Chem. Chem. Phys., 2018, 20, 28623-28633.

7 B. Zhao, G. Shao, B. Fan, W. Zhao and R. Zhang, Phys. Chem. Chem. Phys., 2015, 17, 2531-2539.

8 Z. Wang, P. Zhao, D. He, Y. Cheng, L. Liao, S. Li, Y. Luo, Z. Peng and P. Li, Phys. Chem. Chem. Phys., 2018, 20, 14155-14165.

9 G. Datt, C. Kotabage, S. Datar and A. C. Abhyankar, Phys. Chem. Chem. Phys., 2018, 20, 26431-26442.

10 Y. Wang, Y. C. Du, R. Qiang, C. H. Tian, P. Xu and X. J. Han, Adv. Mater. Interfaces, 2016, 3, 1500684.

11 F. Wu, A. M. Xie, M. X. Sun, Y. Wang and M. Y. Wang, J. Mater. Chem. A, 2015, 3, 14358-14369.

12 R. W. Shu, G. Y. Zhang, X. Wang, X. Gao, M. Wang, Y. Gan, J. J. Shi and J. He, Chem. Eng. J., 2018, 337, 242-255.

13 N. Zhou, Q. D. An, Z. Y. Xiao, S. R. Zhai and Z. Shi, RSC Adv., 2017, 7, 45156-45169.

14 Z. C. Wu, K. Tian, T. Huang, W. Hu, F. F. Xie, J. J. Wang, M. X. Su and L. Li, ACS Appl. Mater. Interfaces, 2018, 10, 11108-11115.

15 H. Q. Zhao, Y. Cheng, H. L. Lv, G. B. Ji and Y. W. Du, Carbon, 2019, 142, 245-253.

16 S. K. Singh, H. Prakash, M. J. Akhtar and K. K. Kar, ACS Sustainable Chem. Eng., 2018, 6, 5381-5393.

17 X. L. Wang, X. Huang, Z. R. Chen, X. P. Liao, C. Liu and B. Shi, J. Mater. Chem. C, 2015, 3, 10146-10153.

18 E. Jayamani, S. Hamdan, M. R. Rahman, M. K. B. Bakri and A. Kakar, J. Appl. Polym. Sci., 2015, 132, 42470.

19 H. Q. Zhao, Y. Cheng, W. Liu, L. J. Yang, B. S. Zhang, L. P. Wang, G. B. Ji and Z. J. Xu, Nano-Micro Lett., 2019, 11, 24. 
20 X. J. Wu, X. T. Fan, S. J. Xie, J. C. Lin, J. Cheng, Q. H. Zhang, L. Y. Chen and Y. Wang, Nat. Catal., 2018, 1, 772-780.

21 Y. H. Zou, X. F. Yang, C. Lv, T. C. Liu, Y. Z. Xia, L. Shang, G. I. Waterhouse, D. J. Yang and T. R. Zhang, Adv. Sci., 2017, 4, 1600262.

22 Z. C. Wu, W. Hu, T. Huang, P. Lan, K. Tian, F. F. Xie and L. Li, J. Mater. Chem. C, 2018, 6, 8839-8845.

23 K. Wang, Y. Chen, R. Tian, H. Li, Y. Zhou, H. Duan and H. Liu, ACS Appl. Mater. Interfaces, 2018, 10, 11333-11342.

24 S. Y. Wang, X. Ke, S. T. Zhong, Y. R. Lai, D. L. Qian, Y. P. Wang, Q. H. Wang and W. Jiang, $R S C A d v$., 2017, 7, 46436-46444.

25 B. Quan, X. H. Liang, G. B. Ji, Y. N. Zhang, G. Y. Xu and Y. W. Du, ACS Appl. Mater. Interfaces, 2017, 9, 38814-38823.

26 J. Feng, Y. Zong, Y. Sun, Y. Zhang, X. Yang, G. Long, Y. Wang, X. H. Li and X. L. Zheng, Chem. Eng. J., 2018, 345, 441-451.

27 F. Y. Wang, Y. Q. Sun, D. R. Li, B. Zhong, Z. G. Wu, S. Y. Zuo, D. Yan, R. F. Zhuo, J. J. Feng and P. X. Yan, Carbon, 2018, 134, 264-273.

28 Y. Jiang, Y. Chen, Y. J. Liu and G. X. Sui, Chem. Eng. J., 2018, 337, 522-531.

29 D. Li, B. Zhang, W. Liu, X. Liang and G. Ji, Dalton Trans., 2017, 46, 14926-14933.

30 P. Wang, L. Cheng, Y. Zhang and L. Zhang, ACS Appl. Mater. Interfaces, 2017, 9, 28844-28858.

31 Y. L. Zhou, J. Muhammad, X. F. Zhang, D. X. Wang, Y. P. Duan, X. L. Dong and Z. D. Zhang, RSC Adv., 2018, 8, 6397-6405.

32 R. Qiang, Y. Du, H. Zhao, Y. Wang, C. Tian, Z. Li, X. Han and P. Xu, J. Mater. Chem. A, 2015, 3, 13426-13434.

33 D. Ding, Y. Wang, X. Li, R. Qiang, P. Xu, W. Chu, X. Han and Y. Du, Carbon, 2017, 111, 722-732.

34 S. Dai, Y. Cheng, B. Quan, X. Liang, W. Liu, Z. Yang, G. Ji and Y. Du, Nanoscale, 2018, 10, 6945-6953.

35 W. Chu, Y. Wang, Y. Du, R. Qiang, C. Tian and X. Han, J. Mater. Sci., 2017, 52, 13636-13649.

36 J. Li, L. Wang, D. Zhang, Y. Qu, G. M. Wang, G. Tian, A. H. Liu, H. J. Yue and S. H. Feng, Mater. Chem. Front., 2017, 1, 1786-1794.

37 G. Li, L. Wang, W. Li and Y. Xu, Microporous Mesoporous Mater., 2015, 211, 97-104.

38 Y. Du, T. Liu, B. Yu, H. Gao, P. Xu, J. Wang, X. Wang and X. Han, Mater. Chem. Phys., 2012, 135, 884-891.

39 L. Wang, X. Jia, Y. Li, F. Yang, L. Zhang, L. Liu, X. Ren and H. Yang, J. Mater. Chem. A, 2014, 2, 14940-14946.

40 H. L. Xu, X. W. Yin, M. Zhu, M. K. Han, Z. X. Hou, X. L. Li, L. T. Zhang and L. F. Cheng, ACS Appl. Mater. Interfaces, 2017, 9, 6332-6341.

41 W. Feng, Y. M. Wang, J. C. Chen, B. Q. Li, L. X. Guo, J. H. Ouyang, D. C. Jia and Y. Zhou, J. Mater. Chem. C, 2018, 6, 10-18.

42 H. B. Zhao, Z. B. Fu, H. B. Chen, M. L. Zhong and C. Y. Wang, ACS Appl. Mater. Interfaces, 2016, 8, 1468-1477.

43 B. Zhao, X. Q. Guo, W. Y. Zhao, J. S. Deng, G. Shao, B. B. Fan, Z. Y. Bai and R. Zhang, ACS Appl. Mater. Interfaces, 2016, 8, 28917-28925.
44 B. Quan, X. H. Liang, G. B. Ji, Y. Cheng, W. Liu, J. N. Ma, Y. N. Zhang, D. R. Li and G. Y. Xu, J. Alloys Compd., 2017, 728, 1065-1075.

45 N. Zhou, Q. D. An, Z. Y. Xiao, S. R. Zhai and Z. Shi, ACS Sustainable Chem. Eng., 2017, 5, 5394-5407.

46 J. L. Lv, S. R. Zhai, C. Gao, N. Zhou, Q. D. An and B. Zhai, Chem. Eng. J., 2016, 289, 261-269.

47 C. Luo, W. Duan, X. Yin and J. Kong, J. Phys. Chem. C, 2016, 120, 18721-18732.

48 M. M. Lu, M. S. Cao, Y. H. Chen, W. Q. Cao, J. Liu, H. L. Shi, D. Q. Zhang, W. Z. Wang and J. Yuan, ACS Appl. Mater. Interfaces, 2015, 7, 19408-19415.

49 Y. Sun, J. Xu, W. Qiao, X. Xu, W. Zhang, K. Zhang, X. Zhang, X. Chen, W. Zhong and Y. Du, ACS Appl. Mater. Interfaces, 2016, 8, 31878-31886.

50 X. Qiu, L. Wang, H. Zhu, Y. Guan and Q. Zhang, Nanoscale, 2017, 9, 7408-7418.

51 P. Xu, X. J. Han, C. Wang, D. H. Zhou, Z. S. Lv, A. H. Wen, X. H. Wang and B. Zhang, J. Phys. Chem. B, 2008, 112, 10443-10448.

52 Y. Song, L. He, X. Zhang, F. Liu, N. Tian, Y. Tang and J. Kong, J. Phys. Chem. C, 2017, 121, 24774-24785.

53 S. S. Gao, Q. D. An, Z. Y. Xiao, S.-R. Zhai and D. J. Yang, ACS Appl. Nano Mater., 2018, 10, 5895-5906.

54 N. Zhou, Q. D. An, W. Zheng, Z. Y. Xiao and S. R. Zhai, RSC Adv., 2016, 6, 98128-98140.

55 L. J. Deng and M. G. Han, Appl. Phys. Lett., 2007, 91, 023119.

56 F. Yan, J. Y. Kang, S. Zhang, C. Y. Li, C. L. Zhu, X. T. Zhang and Y. J. Chen, Nanoscale, 2018, 10, 18742-18748.

57 S. Y. Wang, S. S. Peng, S. T. Zhong and W. Jiang, J. Mater. Chem. C, 2018, 6, 9465-9474.

58 S. K. Singh, M. J. Akhtar and K. K. Kar, ACS Appl. Mater. Interfaces, 2018, 10, 24816-24828.

59 B. Zhao, G. Shao, B. Fan, W. Zhao, Y. Xie and R. Zhang, J. Mater. Chem. A, 2015, 3, 10345-10352.

60 Q. S. Wu, J. W. Liu, G. S. Wang, S. F. Chen and S. H. Yu, Sci. China Mater., 2016, 59, 609-617.

61 S. S. Dai, B. Quan, B. S. Zhang, X. H. Liang and G. B. Ji, Dalton Trans., 2018, 47, 14767-14773.

62 L. Guo, S. S. Gao, Q. D. An, Z. Y. Xiao, S. R. Zhai, D. J. Yang and L. Cui, RSC Adv., 2019, 9, 766-780.

63 A. M. Xie, F. Wu, W. C. Jiang, K. Zhang, M. X. Sun and M. Y. Wang, J. Mater. Chem. C, 2017, 5, 2175-2181.

64 Q. Hu, X. Qi, H. Cai, R. Xie, L. Long, Z. Bai, Y. Jiang, S. Qin, W. Zhong and Y. Du, Sci. Rep., 2017, 7, 1-12.

65 H. M. Zhang, C. L. Zhu, Y. C. Chen and H. Gao, ChemPhysChem, 2014, 15, 2261-2266.

66 L. Liu, Z. D. He, Y. T. Zhao, J. C. Sun and G. X. Tong, J. Alloys Compd., 2018, 765, 1218-1227.

67 B. Zhao, W. Zhao, G. Shao, B. Fan and R. Zhang, ACS Appl. Mater. Interfaces, 2015, 7, 12951-12960.

68 H. T. Guan, H. Y. Wang, Y. L. Zhang, C. J. Dong, G. Chen, Y. D. Wang and J. B. Xie, Appl. Surf. Sci., 2018, 447, 261-268.

69 H. Q. Zhao, Y. Cheng, J. N. Ma, Y. N. Zhang, G. B. Ji and Y. W. Du, Chem. Eng. J., 2018, 339, 432-441. 